Disclosure of Interests: M. Begoña Ruiz-Argüello Employee of: Progenika Biopharma - Grifols employee, Ainara Maguregui Employee of: Progenika Biopharma - Grifols employee, Antonio Martínez Employee of: Progenika Biopharma - Grifols employee, Daniel Nagore Employee of: Progenika Biopharma - Grifols employee

DOI: 10.1136/annrheumdis-2019-eular.2373

\section{AB0405 ADALIMUMAB THERAPEUTIC DRUG MONITORING TEST VALIDATED FOR MEASURING ABP 501 BIOSIMILAR}

M. Begoña Ruiz-Argüello, Ainara Maguregui, Antonio Martínez, Daniel Nagore. Progenika Biopharma - Grifols, RandD, Derio, Spain

Background: Promonitor ${ }^{\circledR}-\mathrm{ADL}$ test is routinely used to monitor IBD patients treated with adalimumab (ADL). ABP 501 (adalimumab biosimilar; Amgen) was authorised throughout the European Union in March 2017 and has been recently launched in several countries. Therapeutic drug monitoring (TDM) is broadly used as an aid for patient management. However, all TDM tests available should be properly validated against each new approved biosimilar in order to ensure safe application for patient monitoring as these may guide dose adjustments.

Objectives: Here we validate the suitability and performance of Promonitor-ADL CE-marked test for quantification of the adalimumab biosimilar ABP 501 in comparison to the reference adalimumab drug (Abbvie).

Methods: The validation study was in accordance with the design requirements established in the Clinical \& Laboratory Standards Institute (CLSI) guideline EP17-A2 (Lower Limit of Quantification, LLOQ) and EP10-A3 (imprecision and bias). CLSI guidelines set a standard for the diagnostic industry accepted by all regulatory agencies. LLOQ was determined with four independent human serum sample matrices per each of three low level ADL concentrations, replicated three times per two lots of Promonitor-ADL (Progenika, Spain) kits for each drug, the reference drug and the adalimumab biosimilar ABP 501, over three days by one operator. Imprecision was evaluated using three replicates of five human serum sample matrices representative of clinically relevant $A D L$ concentrations and spanning the measurement range of Promonitor-ADL, run on one instrument with one kit lot by one operator over six non-consecutive operating days and one run per testing day, with an acceptance criteria of $\mathrm{CV} \% \leq 20 \%$.

Results: The LLOQ of Promonitor-ADL for the adalimumab biosimilar ABP 501 and reference adalimumab were $0.34 \mathrm{mg} / \mathrm{mL}$ and $0.36 \mathrm{mg} / \mathrm{mL}$, respectively. LLOQ values met accuracy goal proposed based on total error $\leq 25 \%$ and precision. The imprecision of Promonitor-ADL calculated by estimating the components of variance due to within-run and betweenday factors meet the accuracy goals proposed at all concentration levels of $\mathrm{ABP} 501$ vs the reference adalimumab (CV\% between $5 \%$ and $10 \%$ ). The bias study showed that Promonitor-ADL can equally measure the active moiety $A D L$ either in the reference biologic $A D L$ or in the biosimilar ABP 501. The test is able to quantify the adalimumab biosimilar ABP 501 in the measurement range of 0.9 to $10.9 \mathrm{mg} / \mathrm{mL}$ with a bias estimate of -0.089 to $0.306 \mathrm{mg} / \mathrm{mL}$ and an overall imprecision of $6 \%$ to $9 \%$. The measurement range includes the recommended clinical decision points.

Conclusion: Promonitor-ADL test can equivalently measure either the reference $A D L$ or the approved adalimumab biosimilar ABP 501 with the same sensitivity, precision and accuracy.

Disclosure of Interests: M. Begoña Ruiz-Argüello Employee of: Progenika Biopharma - Grifols employee, Ainara Maguregui Employee of: Progenika Biopharma - Grifols employee, Antonio Martínez Employee of: Progenika Biopharma - Grifols employee, Daniel Nagore Employee of: Progenika Biopharma - Grifols employee

DOI: 10.1136/annrheumdis-2019-eular.2360

\section{AB0406 THE POTENT WEAPON FOR RHEUMATOID ARTHRITIS- INTERSTITIAL LUNG DISEASE : RITUXIMAB EXPERIENCES}

Burak Karakaş, Mehmet Emin Derin, Fatih Albayrak, Ali Şahin. Sivas Cumhuriyet University, sivas, Turkey

Background: Rheumatoid arthritis (RA) is a common inflammatory disease with unknown etiology and systemic involvement (1). About $40 \%$ of RA patients have extraarticular involvement. Lung involvement is the most common extraarticular finding. The use of rituximab (RTX) in the treatment of rheumatoid arthritis- interstitial lung disease (RA-ILD) has been increasing in recent years (2).

Objectives: To present our rituximab experience in patients with RA-ILD. Methods: Between April 2015 and April 2018, sixteen patients with RAILD who were followed up with RTX treatment in our university internal medicine-rheumatology department were included in this study. High resolution computed tomography (HRCT), carbon monoxide diffusion measurement (DLCO), pulmonary function test (PFT) and routine laboratory tests were examined.

Results: The median age of the patients was 68 years (min: 52-max: 77). 4 patients (25\%) were male and $12(75 \%)$ patients were female. Four of our patients $(25 \%)$ were active smokers. Non-specific interstitial pneumonia (NSIP) was seen in $10(62.5 \%)$ patients and usual interstitial pneumonia (UIP) was seen in $6(37.5 \%)$ patients. Before RTX, 8 patients were receiving methotrexate and 8 patients were using leflunomide. Fou patients had anti-TNF (tumor necrosis factor) treatment. Median during treatment time was 6 months. Other features of the patients are summarized in Table 1. All patients had dyspnea with exertion before treatment The Forced Vital Capacity (FVC) median was\% 70 and DLCO was\% 66 . Although 2/16 patients received cyclophosphamide treatment, there was no clinical response and then RTX treatment was started. Protocol of treatment was every 6 months (days 0 and 15 days 1 g). After 6 months, FVC values improved with NSIP pattern $(p=0.04)$. There was no improvement in the UIP pattern but remained stable (Table 2). Clinically, patients' exertional dyspnea improved. There were no serious side effects in the follow-up of the patients.

Conclusion: There is no valid guideline for RA-ILD treatment. Patientbased decision-making is important in the treatment of these patients. In recent years, RTX seems to be quite effective in RA-ILD. However, longterm and extensive studies are needed in terms of maintenance treatment and possible side effects.

\section{REFERENCES}

[1] Mclnnes IB, G. Schett, The pathogenesis of rheumatoid arthritis. N Engl J Med 2011; 365: 2205-19.

[2] Deborah Assayag, Joyce S. Lee, Talmadge E. Kıng, Jr., Rheumatoid Arthritis Associated Interstitial Lung Disease: A Review, MEDICINA (Buenos Aires) 2014; 72: 158-165

Table 1. Clinical and epidemiological Features of the RA patients

\begin{tabular}{lc}
\hline Age & 68 (min:52-max:77) \\
\hline Gender: Male & $4(25 \%)$ \\
Female & $12(75 \%)$ \\
Serology: Positive & $14(87.5 \%)$ \\
Negative & $2(12.5 \%)$ \\
Smoking history & $4(25 \%)$ \\
Medication: Methotrexate & $8(40 \%)$ patients \\
Leflunomide & $8(40 \%)$ \\
Anti-TNF & $4(20 \%)$ \\
Duration of disease & 10 years \\
Duration of treatment & 6 months \\
IAH pattern: NSIP & $10(62.5 \%)$ \\
UIP & $6(37.5 \%)$ \\
\hline
\end{tabular}

Table 2. The FVC and DLCO values of pre- and post- treatment

\begin{tabular}{lccc}
\hline & UIP & NSIP & \\
\hline Pre FVC & 70 & 76 & \\
Post FVC & 70 & 80 & $\mathrm{p}=0.04$ \\
& $\mathrm{p}>0.05$ & 70 & \\
Pre DLCO & 65 & 71 & $\mathrm{p}>0.05$ \\
Post & 66 & & \\
DLCO & $\mathrm{p}>0.05$ & &
\end{tabular}

Disclosure of Interests: None declared DOI: 10.1136/annrheumdis-2019-eular.7559 